\title{
The development of designated driving application based on Android platform and Ali cloud sever
}

\author{
Xinge LI \\ School of North China Electric Power University, Baoding 071000, China; \\ 122102894@qq.com
}

Keywords: designated driving. Android platform. Ali cloud sever. taxi service

\begin{abstract}
This paper discussed the development process of designated driving application on Android platform, using Android SDK, Eclipse IDE to build and Ali cloud sever for back-stage management, and explained the key technologies of the building of android platform and Ali cloud sever in a further step. By the means of Ali cloud sever, the system successfully provide thoughtful taxi service, perfect payment mode and evaluation function for customers.
\end{abstract}

\section{Android system and Cloud server}

\subsection{Android system architecture}

Android system architecture is divided into four layers on the whole. From the top down, it includes Applications, Application framework, Libraries, Android Runtime and Linux Kernel. The application development of designated driving is based on framework and component. Although android itself provides a number of components in the frame for supply, we also develop a new set of components for the requirement of application.

Android development libraries and running environment includes a set of core libraries, providing most of the functionality in core library of Java language and Dalvik virtual machine. And Linux kernel layer is used to provide the underlying service of system.

From the view of android system architecture, android, compared with other platforms, has its own characteristics. Combining with these features, we make full use of module interface provided by android and tool set of Android SDK offered by Google, which includes compilers, resource manager, the debugger and simulator, for developing distinctive application.

\subsection{Cloud server technology}

Cloud server is a kind of computing service with high efficiency and reliability, meanwhile, its processing capacity can be flexible. And the management mode is more simple and efficient than the physical server. According to these characteristics, we choose Ali cloud server to assist us to build more stable and safe application.

\section{Overall design of the software system}

\subsection{General functional structure of system}

On the basis of the requirement of designated driving application, the general function design of the whole system is carried out as figure 1 shows. 


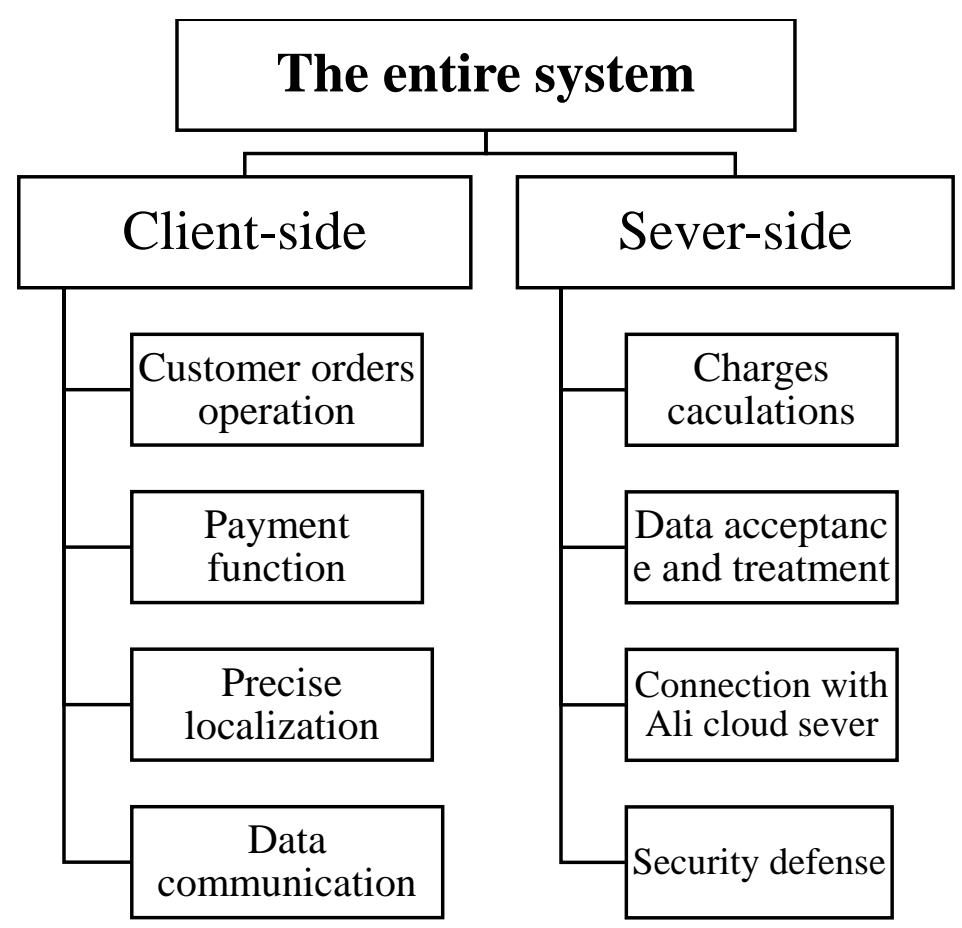

Figure1 general function design of the whole system

- Client-side are mainly concluded by four parts.

Customer orders operation consists of orders establishment, canceling, inquiry and evaluation. Payment function is to ensure a normal trading approach. Precise location is the fundamental part for achieving the whole function. Data communication majors in transporting data to sever.

- Sever-side

Charges calculations is prerequisite for the normal payment. Data acceptance and treatment will analyze the demand for users and execute the corresponding commands.

Connection with Ali cloud is to acquire the information from client-side. Security defense is to ensure the entire system to run safely and prevent being attacked.

\subsection{Cloud server architecture}

The following several aspects shows the key technology of it.

\section{(1) The technique of virtual machine}

In the server virtualization, virtualization software needs to implement many functions, including hardware abstraction, resource allocation, scheduling, management and so on.

\section{(2) data store technology}

As the key technology of cloud sever, it is not only required to satisfy user's demand, but also supply service for customers. Based on these, data store technology should be with the advantages of distribution, high throughput and transmission rate.

\section{(3) Data management technology}

A suitable data management technology is virtual to the operation of whole system. Therefore, how to enhance data update rate and further increase access rate with random is essential to solve at present.

\section{(4) Distributed programming and computing}

In order to make it easier for users to enjoy the services of cloud computing and use the programming model, for achieving a specific purpose, to write a simple program, Distributed programming and computing are necessary for the construction of system. 


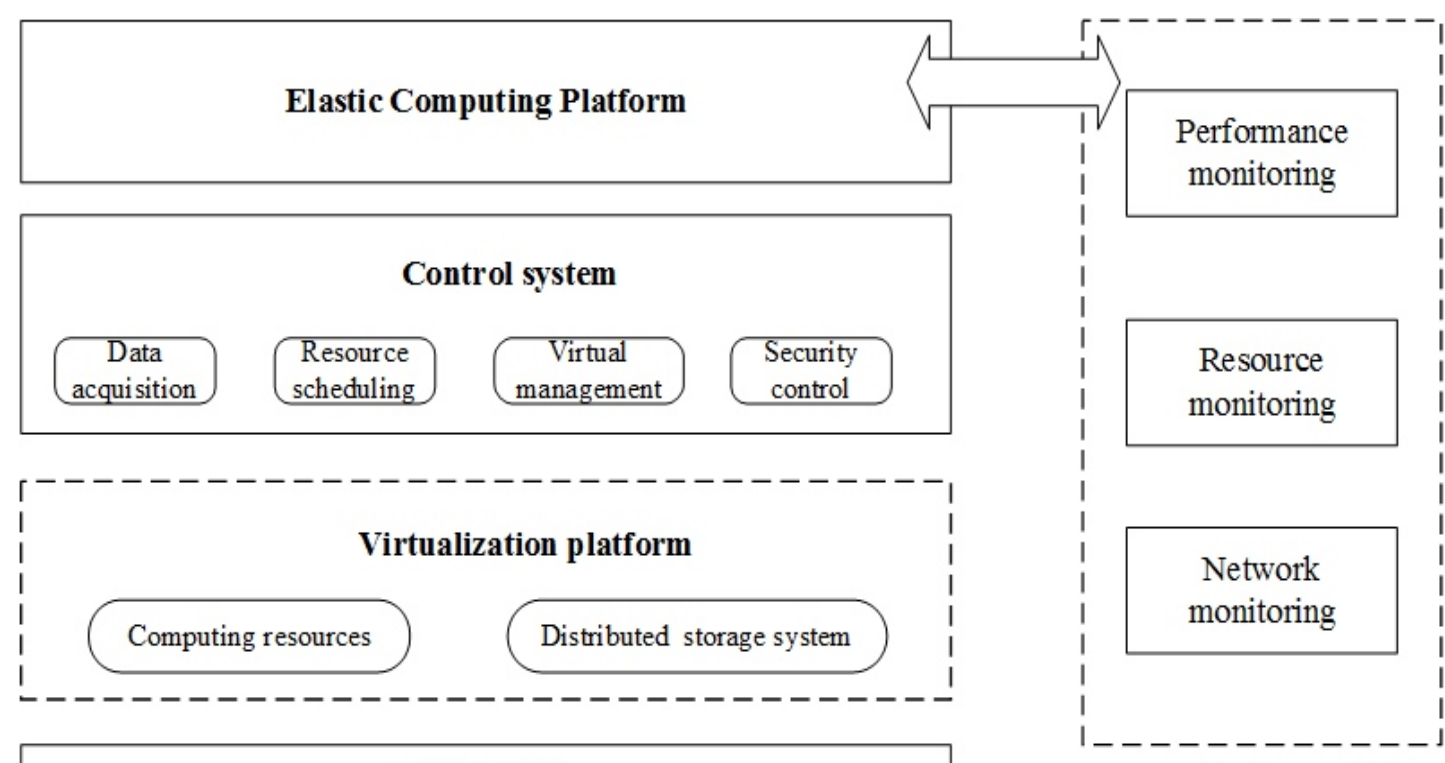

Aliyun OS

Operational monitoring module

Figure2 System architecture of Ali cloud ECS

As we can see from figure 2, Ali cloud system is mainly composed of elastic computing platform, distributed cloud storage platform and operational monitoring module. Compared with traditional server, greatly improve the efficiency of business users.

\section{The technology and implementation of Android system}

For achieving a workable android application, we should know the following points.

\section{- A basic knowledge of Java}

Java is an object-oriented programming language, the entire application code is based on Java. That is to say, a solid foundation of the language determines whether the work can be carried out smoothly.

\section{- The building of android application environment}

We choose eclipse as our development environments for android. Meanwhile, installing Java JDK, android SDK and the ADT plugin as auxiliary development Tools.

\section{- Four major components}

(1) Activity

An application interface containers which can place a variety of controls.

(2) Broadcast Intent Receiver

We can use Broadcast Intent Receiver to respond to an external event.

(3) Service

It is a process running in the background without user interface.

(4) Content Provider

Content Provider is used for data sharing.

\section{- Android virtual machine}

Android virtual machine is an useful product for assisting us to use android environment and develop application. Mastering the android virtual machine technology is important for us to testing the operation of application.

\section{- Application Programming Interface}

From the view of the network, application program interface provides a set of methods for user to send request data, information to application layer so that each layer of network ordinarily responses and finally complete the network data transmission. 


\section{Conclusion}

This paper develop a designated driving application based on the Android platform, in the meantime, introduce the cloud computing technology of Ali cloud sever for massive users. Many computing resources form a dynamic stretching the resource pool through virtualization technology. It not only has local recognition function, but also can access the network by a cloud server with high-rate, which greatly reduce the processing burden of the end customer.

\section{References}

[1] ZHANG Wei-Feng, GUAN Wen-Qiang, LIU Pei-Mei, WANG Na, Electronic Dictionary Based on Android Platform and Cloud Computing. Computer Systems \& Applications. Vol. 24 (2015) No. 2, p. 108-110.

[2] LIU Bin, NI Ming, Design and Implementation of Management System for ICCS-PW Cloud Server. Computer Engineering. Vol. 38 (2012) No. 11, p. 24-26.

[3] FENG Yu-ping, WANG Shu-guang, Android-based Google Map Location Software. JISUANJI YU XIANDAIHUA. (2015) No. 2, p.12-18.

[4] Dissertation class: (Li HaiFeng: Design and implementation of application platform based on software service of Cloud Computing (master, Sun Yat-Sen University, China 2013).p.8.)

[5] Dissertation class: (Lin Xue: Research and Implementation of Cloud Server Management System on IOS Platform (master, Donghua University, China 2014).p.9.)

[6] Dissertation class: (Wang Yan: Design and development of Driving Service System (bachelor, South China Agricultural University, China 2014).p.9.)

[7] Information on http://www.apkbus.com/ 